\title{
Young People in the United Kingdom and Ireland with Perinatally Acquired HIV: The Pediatric Legacy For Adult Services
}

\author{
Caroline Foster, MBBS, MRCPCH, ${ }^{1}$ Ali Judd, M.Sc., Ph.D., ${ }^{2}$ Pat Tookey, M.Sc., Ph.D., ${ }^{3}$ \\ Gareth Tudor-Williams, MBBS, MRCPCH, ${ }^{4}$ David Dunn, M.Sc., Ph.D., ${ }^{2}$ \\ Delane Shingadia, MBBS, MRCPCH,${ }^{5}$ Karina Butler, MBBS, MRCPCH, ${ }^{6}$ \\ Mike Sharland, MBBS, FRCPCH, M.D., ${ }^{7}$ Di Gibb, MBBS, FRCPCH, Ph.D., ${ }^{2}$ \\ and Hermione Lyall, MBBS, MRCPCH, M.D., ${ }^{1}$ \\ on behalf of the Collaborative HIV Paediatric Study (CHIPS)
}

\begin{abstract}
Children with perinatally acquired HIV-1 infection are surviving into adolescence and increasingly transitioning toward adult services. Planning appropriate services in adult life requires an understanding of their progress through pediatric care. We describe the demographic features, disease progression, antiretroviral therapy (ART), and resistance in young people aged 10 years or more living in the United Kingdom and Ireland reported to the National Study of HIV in Pregnancy and Childhood (NSHPC) with prospective annual follow-up in the Collaborative HIV Paediatric Study (CHIPS) between 1996 and September 2007. Six hundred fifty-four perinatally infected young people were identified; $76 \%$ black African, $57 \%$ born abroad. Median age at presentation and duration of follow-up was 1 and 11 years, respectively, if born in the United Kingdom/Ireland, and 8 and 5 years if born elsewhere. One hundred sixty-nine (26\%) ever had an AIDS-defining illness. Ten died during adolescence. At last follow-up, $64 \%$ were on ART, 18\% off treatment having previously received ART and $18 \%$ were ART naive. Of 518 who had received highly active antiretroviral therapy (HAART), 47\% were triple class experienced. At last follow-up $77(12 \%)$ had CD4 counts less than 200 per microliter; of those on HAART, 78\% had HIV-1 RNA $\leq 400$ copies per milliliter, median CD4 count 554 (interquartile range [IQR] 324-802). Among 166 with resistance assays on HAART, $52 \%$ and $12 \%$ had dual- and triple-class HIV-1-associated resistance mutations, respectively. One hundred three $(16 \%)$ young people had transferred to adult services. Young adults with perinatally acquired HIV-1 infection require coordinated multidisciplinary transitional care services and careful long-term follow-up in adult life.
\end{abstract}

\section{Introduction}

I EUROPE, prior to the availability of antiretroviral therapy (ART), half of HIV-infected children died by their tenth birthday and the majority of survivors were symptomatic. ${ }^{1}$ The introduction of highly active antiretroviral therapy (HAART) in 1996 has resulted in a substantial increase in life expectancy for adults with HIV infection living in high- income countries. ${ }^{2,3}$ Additionally, in pediatric populations, HAART has been associated with a substantial reduction in HIV-associated mortality and morbidity. ${ }^{4-6}$

Consequently, children with perinatally acquired HIV infection are now surviving into adolescence and early adult life and increasingly transitioning from pediatric to adult services. While transitional care for adolescents with HIV is an emerging specialty, much can be learned from the experiences

\footnotetext{
${ }^{1}$ Imperial College Healthcare NHS Trust, London, United Kingdom.

${ }^{2}$ MRC Clinical Trials Unit, London, United Kingdom.

${ }^{3}$ UCL Institute of Child Health, London, United Kingdom.

${ }^{4}$ Imperial College Department of Paediatrics, London, United Kingdom.

${ }^{5}$ Great Ormond Street Hospital for Children, London, United Kingdom.

${ }^{6}$ Our Lady's Hospital for Sick Children, Dublin, Ireland.

${ }^{7}$ St. George's Hospital, London, United Kingdom.
} 
of other specialties caring for young people surviving with chronic diseases of childhood in establishing optimal care pathways. Studies have shown poor attendance and adherence among some cohorts of young people surviving other chronic conditions of childhood, including cystic fibrosis, diabetes, and congenital heart disease, resulting in increased morbidity and mortality following transfer to adult services. ${ }^{7-10}$ Initial data suggest poorer adherence to ART resulting in lower rates of virologic suppression in HIV-infected adolescents compared with younger pediatric and adult cohorts. ${ }^{11,12}$ Additionally, younger age is associated with lower rates of uptake of outpatient care in adults with sexually acquired HIV and there are already concerns regarding attendance rates in young people with perinatally acquired HIV following transfer to adult services. ${ }^{13,14}$ In recognition of the poor service provision for adolescents with severe chronic diseases the Department of Health in the United Kingdom has clear guidelines recommending pathways to facilitate optimal transitional care across all specialties ${ }^{15}$ and HIV specific guidelines are available from the Children's HIV Association. ${ }^{16}$

Planning future service provision for young adults with perinatally acquired HIV infection requires an understanding of their progression through pediatric care. We describe the mortality, morbidity, immunologic, and virologic responses to antiretroviral therapy and HIV-1-associated resistance mutations in a cohort of vertically HIV-infected young people, surviving into adolescence and transitioning toward adult services in the United Kingdom or Ireland.

\section{Methods}

\section{Study design}

The Collaborative HIV Paediatric Study (CHIPS) has been described in detail elsewhere. ${ }^{4}$ Briefly, all children with known perinatal exposure to HIV, or diagnosed with HIV under the age of 16 in the UK or Ireland, are reported to the National Study of HIV in Pregnancy and Childhood (NSHPC). Detailed clinical, laboratory, and treatment information is then collected annually through CHIPS for almost all ( $>90 \%$ by 2007) infected infants and children. By September 30, 2007, 1313 vertically infected children had been included in CHIPS, of whom 654 were aged 10 years or older at last report $(96 \%$ seen in 2005-2007) and comprise the cohort analyzed here. CHIPS and the NSHPC have ethics approval from the London MREC, including approval for linkage with the U.K. HIV Drug Resistance Database.

\section{Statistical methods}

All analyses were conducted using Stata 9 (Stata Corp, College Station, TX). Demographic differences were explored using descriptive statistics, with particular focus on place of birth, age at and year of presentation, and age at last followup. Proportions were compared using $\chi^{2}$ tests, and medians and means were compared using two-sample Wilcoxon ranksum tests and $t$ tests, respectively. HAART was defined as three or more antiretroviral drugs from two or more classes or a nucleoside reverse transcriptase inhibitor (NRTI) regimen containing abacavir. Clinical events were defined according to the Centers for Disease Control classification system. ${ }^{17}$ Weight and height $z$ scores were compared to normative U.K. data ${ }^{18}$ with severe growth retardation defined as a weight-forage and/or height-for-age $z$-score less than -2 .
Resistance test results were obtained by matching the CHIPS cohort to the U.K. HIV Drug Resistance Database, using a combination of hospital number, hospital of care, date of birth, and patient initials. Multiple results for a single individual are presented as cumulative mutations. ${ }^{19}$ Resistance was defined as one or more major mutations as specified in the latest IAS-USA guidelines, ${ }^{20}$ plus selected additional mutations (in reverse transcriptase, any mutation at G190 or T215; in protease, V32I and I47V/A in combination, or seven or more minor lopinavir mutations). As in other analyses, ${ }^{19}$ because the lamivudine/FTC-associated M184V mutation confers no significant resistance to other NRTIs it was considered as a fourth class of mutation.

\section{Results}

\section{Demographic characteristics}

Table 1 shows the demographic characteristics of the 654 young people aged 10 years or older at last follow-up, by age at last follow-up and country of birth. Just over half (53\%) were female, three quarters $(76 \%)$ were black African, and $57 \%$ were born abroad. Sixty percent of those born in the U.K./Ireland were of black African origin, compared to $88 \%$ of those born abroad $\left(\chi^{2} 2, p<0.001\right)$. Age at presentation differed by place of birth: median age at presentation of those born in the U.K./Ireland was 1 year, compared to 8 years for those born abroad $(p<0.001)$, and the median duration of follow-up was 11 and 5 years, respectively $(p<0.001)$. Almost a quarter $(22 \%)$ of children born in the U.K./Ireland were identified prospectively from birth compared to $2.5 \%$ of those born abroad, while the proportion identified on screening following the diagnosis of a family member was higher in those born abroad ( $41 \%$ and $31 \%$, respectively, $\left.\chi^{2} 5, p<0.001\right)$.

\section{AIDS diagnoses and mortality}

A quarter $(169,26 \%)$ had ever had an AIDS diagnosis (CDC stage C). Sixty-one (9\%) presented with AIDS, but there was no difference by age at presentation $(9 \%$ for less than 10 years versus $12 \%$ for 10 or more years, $\left.\chi^{2} 1, p=0.234\right)$. A third $(32 \%$, 90) of those born in the U.K./Ireland had been diagnosed with at least one AIDS defining illness, compared with $24 \%$ (89) of those born elsewhere $\left(\chi^{2} 1, p=0.030\right)$. AIDS diagnoses for the whole cohort included Pneumocystis carinii pneumonia/ cytomegalovirus (PCP/CMV; 6\%, 41), other opportunistic infections $(7 \%, 48)$, failure to thrive $(11 \%, 70)$, serious recurrent bacterial infections $(10 \%, 67)$, HIV encephalopathy $(4 \%$, $26)$ and cancer $(2 \%, 11)$.

Ten children died after their tenth birthday (Table 2), of whom nine were born abroad and five first presented prior to the introduction of HAART in 1997. Six were aged 10 years or older at presentation, five had relatively short follow up periods ( $<13$ months) until death, and for six CD4 count prior to death was less than 200 cells per milliliter. Nine died of causes attributable to HIV infection, and the tenth died with pancreatitis while on a HAART combination, which included stavudine and didanosine.

\section{Antiretroviral therapy}

Overall, at last follow-up, almost one in five $(18 \%, 117)$ young people had never received ART (Fig. 1). ART initiation differed by age at presentation: nearly a quarter $(24 \%, 35 / 143)$ 


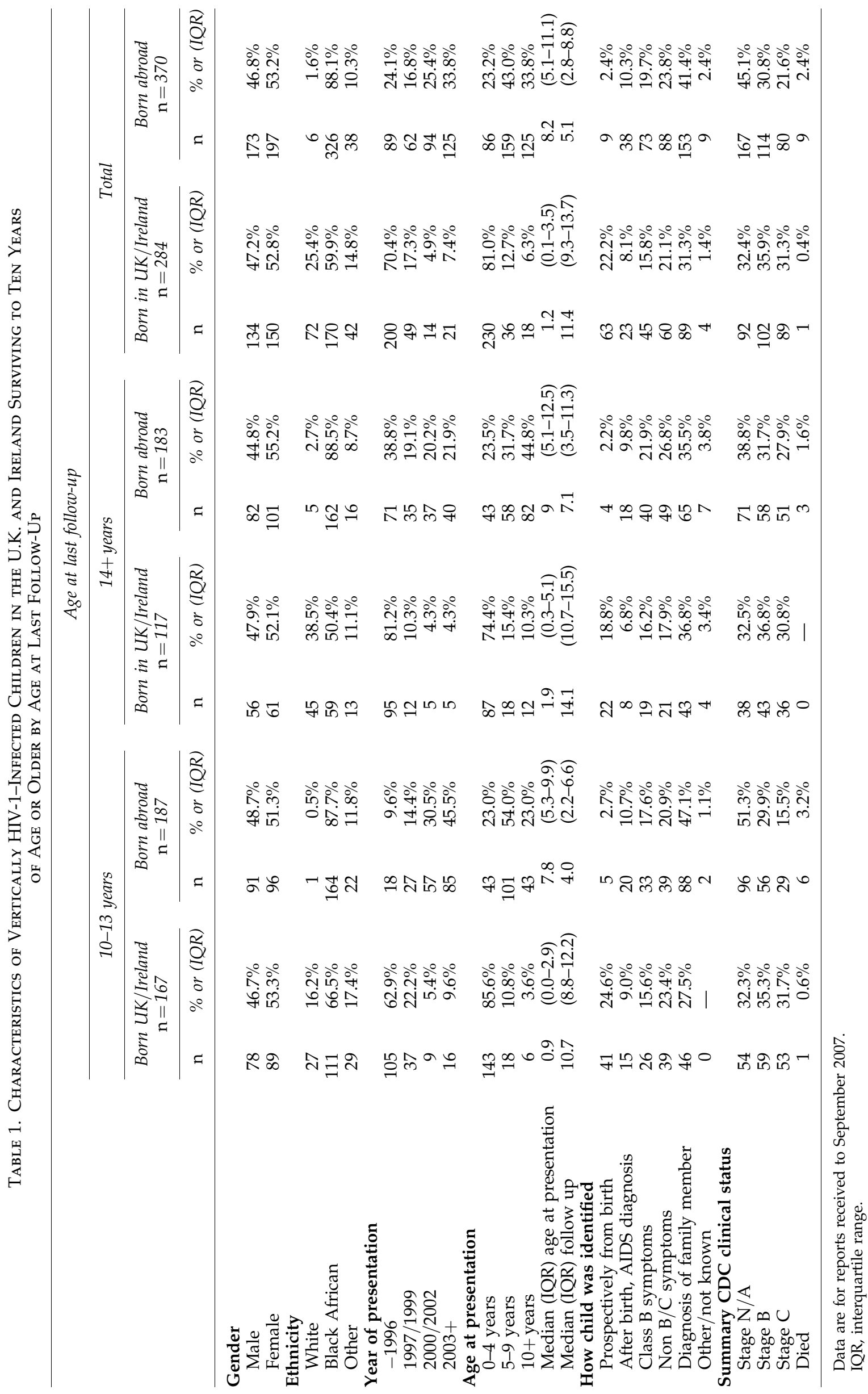




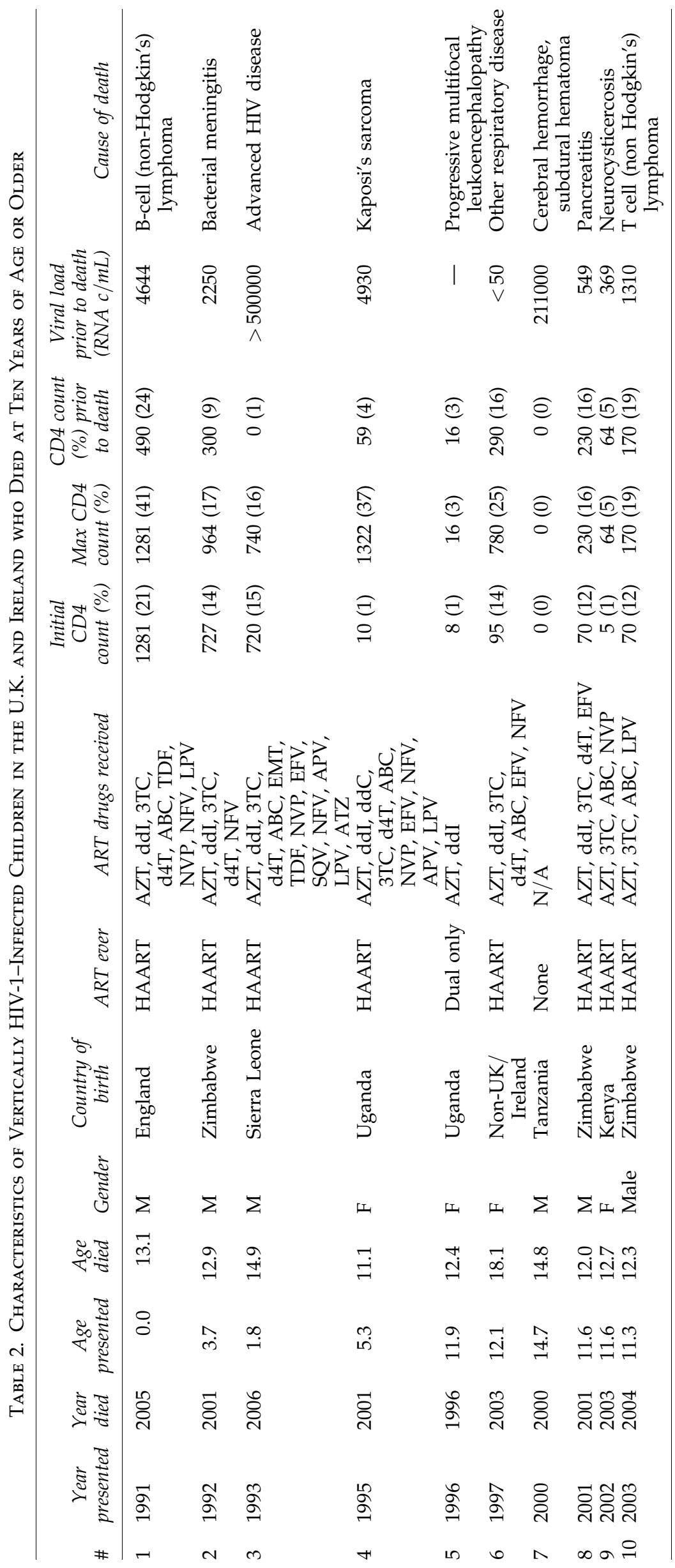




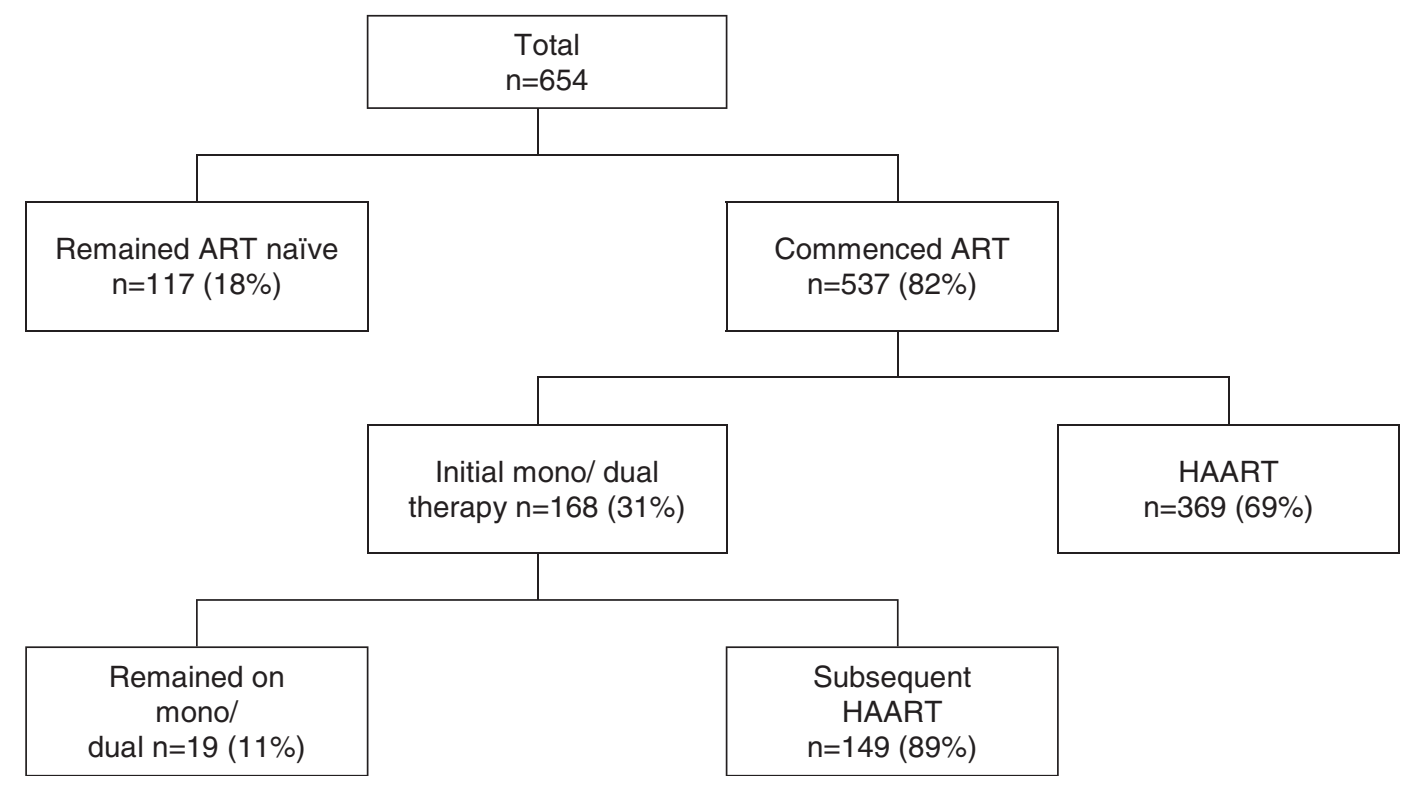

FIG. 1. Antiretroviral treatment history of vertically HIV-infected children in the United Kingdom and Ireland surviving to age 10 years or more.

of those presenting at age 10 years or older remained ART naive, compared to $16 \%(82 / 511)$ of those presenting 10 years or younger $\left(\chi^{2}, p=0.020\right)$. Nineteen had only received monoor dual ART regimens, of whom nine were still in active follow-up in 2005-2007: of these, only one remained on dual therapy, with eight having stopped all ART. The remaining children were lost to follow up (4), had transferred to adult care (5), or had died (1).

Of the 518 adolescents who had received three or four drug ART (HAART), 29\% (149) started on mono- or dual therapy, most (89\%) prior to 1998, transferring to HAART a median of 2 years (IQR 1.2-4.7) later. Three quarters $(78 \%, 396)$ of those starting HAART were still receiving HAART at last followup, 19\% (100) had stopped all ART, and 3\% were on mono- or dual regimens. Reasons for stopping HAART included poor compliance/child refusal (49), structured treatment interruption (25), and other (26).

The composition of initial HAART regimens changed over time: prior to 2000 , two thirds $(67 \%)$ of initial HAART regimens contained a protease inhibitor, most frequently nelfinavir (86\%), and 33\% an NNRTI, most commonly nevirapine (91\%). From 2003 onwards, 86\% on initial regimens were NNRTI-based, almost three quarters (73\%) of which contained efavirenz with all initial protease inhibitor-based regimens $(14 \%)$ containing ritonavir-boosted lopinavir. Additionally, $66 \%$ of initial regimens prior to 2000 contained the NRTI stavudine, declining to $9 \%$ for 2003 onwards. Half $(47 \%)$ of those who had ever received HAART were triple class experienced; $85 \%$ had taken non-nucleoside reverse transcriptase inhibitors (NNRTIs) and 60\% protease inhibitors (PIs). Eight young people had ever taken the fusion inhibitor enfuvirtide (T20), and six continued on this drug at last follow-up. Prior to September 2007 no young person had received an integrase or CCR5 inhibitor, or the newer generation PI darunavir or NNRTI etravirine.

\section{Immunology and virology}

Median CD4 count and percentage at HIV diagnosis (at median [IQR] age 5 [0-16] years) were 540 cells per microliter (IQR 241-935) and 20\% (IQR 12\%-28\%), respectively. For those ever taking HAART (including those with previous mono-/dual therapy) CD4 count was 250 per microliter and percentage 13\% (IQR 106-490,6\%-20\%), respectively, at the start of HAART ( $n=518$, median [IQR] age $9(0.5-18)$ years), increasing to 459 and $20 \%$ (IQR 268-770, 14\%-28\%) at 6 months, and 569 (IQR 343-830) and 25\% (IQR 17\%-31\%) at 12 months. Fifty-eight percent achieved virologic suppression 400 copies or less per milliliter 6 months after HAART initiation and $55 \%$ after 12 months. Of those on HAART at last follow-up, $78 \%$ had a viral load 400 copies or less per milliliter and median CD4 cell count and percentage were 555 per microliter (IQR 324-802) and 27\% (IQR 19\%-34\%), respectively. Of those who remained HAART naive at last follow-up (117, median [IQR] age 13 [10-19] years), 7\% had a viral load 400 copies or less per milliliter and median CD4 cell count and percentage were 474 per microliter (IQR 330-600) and 23\% (IQR 17\%-29\%).

At last follow-up 77 (12\%) young people had evidence of severe immunosuppression (CD4 count $<200$ per microliter). Fifty (70\%) were on HAART, 13 were off ART after previously being on HAART, 7 were either ART-naive (3) or on monotherapy (3-atazanavir/ritonavir [1]; lopinavir/ritonavir [1]; ritonavir [1]) or dual therapy (2-tenofovir with atazanavir/ ritonavir [1]; tenofovir with lopinavir/ritonavir [1]) and 6 died (see above).

\section{Resistance}

A total of 423 resistance test results were available for 211 $(32 \%)$ of the 654 young people. The most common HIV subtypes were C (77), A (49), B (26), and D (25). 
Thirty-six of the 211 had baseline resistance testing of whom 34 had wild-type virus and two had resistance mutations (patient $1-62 \mathrm{~V}, 65 \mathrm{R}, 106 \mathrm{M}, 115 \mathrm{~F}, 184 \mathrm{~V}$; 2-41L, 74V, $184 \mathrm{~V}, 190 \mathrm{~A}, 210 \mathrm{~W}, 215 \mathrm{Y})$. A further 9 had a resistance test after commencing mono- or dual therapy but prior to HAART; 4 had wild-type virus and $5(56 \%)$ had resistance mutations (TAMS [5] 184I [1], 69S [1]). Of the 7 patients with resistance mutations, $5 \mathrm{e}$ had presented 2 years of age or older, and none received mother-to-child prophylaxis.

One hunded sixty-six (79\%) had their first resistance test after the start of HAART, with a median of 3 years (IQR 1-5) between HAART initiation and the first resistance assay. Cumulative results indicated that $23(14 \%)$ had wild-type virus, 81 (49\%) had M184I/V mutations, and 95 (57\%) had non-M184V NRTI mutations, most frequently TAMS (RT positions 41L [48], 67N [46], 70R [28], 210W [31], 215any [88], and 219E/Q [27]). One hundred eight (65\%) had NNRTIassociated mutations, most commonly $103 \mathrm{~N}$ (55), 181C/I (45) and 190A (27). Major protease mutations were detected in 43 (26\%) children, most commonly 90M (19) and 46I/L (14).

One hundred thirty-eight $(65 \%)$ of the 211 were triple class experienced of whom $53 \%$ and $16 \%$ had dual- and triple-class HIV-1-associated resistance mutations.

\section{Growth}

Mean height $z$ score at first measurement was -0.8 (standard deviation $[\mathrm{SD}]=1.3$ ) from published norms ${ }^{18}$ and was slightly lower among those first measured at age 10 years or older compared to younger than 10 years $(-1.1, n=160$ versus $-0.7, n=489$ respectively, $p<0.001$ ). Similarly mean weight $\mathrm{z}$-score at first measurement was $-0.5(\mathrm{SD}=1.3)$, and was marginally lower among those first measured at age 10 or more compared to younger than 10 years $(-0.7, n=159$ versus $-0.4, n=493$, respectively, $p=0.022$ ). Sixteen (3\%) of those first measured at younger than 10 years had severe growth retardation, and similarly 11 (9\%) measured for the first time at 10 years or older. At last growth measurement, 13 of 288 young people $(5 \%)$ aged $\geq 13$ had evidence of severe growth retardation all of whom were on HAART.

\section{Transfer to adult care}

One hundred three $(16 \%)$ of the young people had transferred to adult services by September 30, 2007, at a median age of 17 years (range, 15-22). They had been followed in CHIPS for a median of 12 years (IQR 7-15), longer for those born in the U.K./Ireland (15 years [IQR 12-17]) than for those born abroad (9 years [IQR.5-13]). Thirty-nine (38\%) had ever had an AIDS diagnosis by the time of transfer to adult care. Sixteen were ART-naïve at transfer and of the 82 who had ever received HAART, half (52\%) were triple-class experienced.

At point of transfer to adult services a quarter (28) had evidence of severe immunosuppression (CD4 count $<200$ per microliter). Sixty were receiving HAART, for whom median CD4 count at last measurement was 318 per microliter (IQR 179-576) and 33 (55\%) had viral loads less than 400 copies per milliliter. Resistance test results were available for 41 of the 103 young people, and 17 had dual- and 6 tripleclass resistance; $15(65 \%)$ of the 23 with dual- or triple-class resistance had prior mono-/dual therapy before starting HAART.

\section{Discussion}

This study shows that children with perinatally acquired HIV infection living in the United Kingdom or Ireland and followed in the CHIPS cohort are not only surviving into adolescence but also transferring to adult services. High uptake of antenatal testing ${ }^{21}$ reduced transmission rates from diagnosed women, ${ }^{22}$ improved survival following HAART and later age at presentation among those born abroad ${ }^{6}$ mean that the average age of perinatally infected children and young people in the United Kingdom or Ireland continues to rise, with half of the CHIPS cohort now aged 10 years or over. By September 2007, 8\% of the entire CHIPS cohort had already entered adult services. All of the 654 young people described in this paper were aged 10 years or over at last follow-up and therefore all will require adult services by the end of 2015, assuming that transfer continues at a median age of 17 years. Furthermore, this group will grow in size as additional young people are newly diagnosed in adolescence over the coming years. Recent studies illustrate the acceptability and feasibility of HIV screening for adolescents within the emergency department ${ }^{23}$ and the promotion screening all offspring of adults living with $\mathrm{HIV}^{24}$ may further enhance diagnoses in adolescence. Over one fifth of this cohort was initially diagnosed aged over 10 years, the majority of whom were born abroad. The current trend for dispersal of migrants throughout the United Kingdom will increase the number of health professionals caring for young adults with perinatally acquired HIV.

Many young people in this cohort have complex treatment histories, with four fifths (79\%) having been exposed to HAART, with disappointing levels of viral suppression overall. This may be accounted for, in part, by unavoidable prior exposure to suboptimal mono- and dual therapy in the preHAART era, seen in a third of this cohort. Additionally, suboptimal adherence during the turbulent period of adolescence ${ }^{25}$ is well recognized both in HIV and other chronic diseases ${ }^{26,27}$ resulting in impaired response to treatment with increased morbidity and mortality. Almost one tenth of the young people who had ever started HAART were currently off therapy due to poor adherence/refusal. In young people with poor adherence, the balance between maintaining current immunologic function and clinical health, against the risk of acquisition of resistance mutations on failing regimens that will limit future treatment options is complex. In a U.S. study, almost two thirds of adolescents maintained on initial nonsuppressive HAART regimens developed drug resistance mutations and this was more likely to occur on NNRTI- than protease inhibitor-based HAART, and may influence prescribing in naive patients. ${ }^{28}$

Maintaining adherence is particularly difficult in an adolescent population on HAART, half of whom are triple-class experienced and often do not have once-daily low pill burden options available. Half of those who had ever taken HAART were triple-class experienced, and of those who had resistance assays two thirds had evidence of dual- or triple-class resistance. Some young people will therefore require new agents, both in existing as well as novel classes of ART, ${ }^{29}$ highlighting the need for equal access to new therapies and adequate pharmacokinetic data for pediatric and adolescent populations when compared to adult populations. Baseline resistance testing prior to HAART is of increasing importance in a 
population, many of whom are born in sub-Saharan Africa and following the successful role out of ART may have received, but be unable to give a history of, antiretroviral agents either in earlier childhood or perinatally in the prevention of mother to child transmission of HIV. Reassuringly, albeit with need for improvement, $78 \%$ of young people in the cohort currently on HAART had a viral load below 400 copies per milliliter with good immune reconstitution (median CD4 555 per microliter) with rates of virologic suppression comparable or better than those reported in perinatally infected cohorts of children and adolescents from Europe and the United States. ${ }^{30,31}$ Research on new strategies for giving HAART need evaluating, including various forms of structured treatment breaks in older children and adolescents, aimed at maximizing adherence and efficacy while on HAART, at the same time minimizing risk of any disease progression or development of resistance.

Future adolescents with perinatally acquired HIV who start HAART with no previous ART exposure are expected to have better virologic control. However, the duration of therapy may be longer, particularly in light of recent evidence supporting earlier initiation of HAART both in infants ${ }^{32}$ and adults. ${ }^{33,34}$ While the medium- and long-term side effects of ART are increasingly recognized in adult populations, ${ }^{35}$ a limitation of this paper is the lack of toxicity data in a population widely exposed to ART throughout postnatal growth and development. This is currently being addressed in CHIPS by prospective data collection on lipodystrophy, lipid profiles, bone density, and pubertal development. However, follow-up in CHIPS ceases when adolescents transfer to adult services and so discussions are underway as to how best to facilitate long-term data collection in this important cohort, with key research areas including growth and puberty, ART-related toxicity, disease progression, fertility, secondgeneration mother-to-child transmission, and neurologic and psychosocial functioning. The lack of neurodevelopmental and psychosocial data, including the impact of adolescence on adherence, is an important limitation of the study in a population by definition born into a family living with HIV. Some of the young people will have lost parents to HIV-related disease, half were born outside the United Kingdom, and the majority are from diverse ethnic minority backgrounds. ${ }^{36}$ In addition, while the impact of HIV-associated encephalopathy in infancy resulting in hypertonic diplegia and early cognitive impairment on mobility and employment in adult life may be predicted $^{37}$ more widespread data on neurocognitive functioning, education and, increasingly as the cohort age, employment, are required.

\section{Conclusion}

The success of HAART has seen perinatally acquired HIV become a chronic disease of childhood. Increasing numbers of young people living with HIV will transfer to adult services in the United Kingdom or Ireland during the next decade. The varied and complex needs of these young people must be met by the development of multidisciplinary transitional care services between pediatric and adult health care providers. The legacy of their pediatric care will continue to impact in adult life and careful long-term follow-up of this unique cohort is required to improve outcomes for young people with HIV in the future.

\section{Author Disclosure Statement}

No competing financial interests exist.

\section{References}

1. Gray L, Newell ML, Thorne C, Peckham C, Levy J. Fluctuations in symptoms in human immunodeficiency virusinfected children: The first 10 years of life. Pediatrics 2001; 108:116-112.

2. Antiretroviral Therapy Cohort Collaboration. Life expectancy of individuals on combination therapy in high-income countries: A collaborative analysis of 14 cohort studies. Lancet 2008;372:293-299.

3. Bhaskaran K, Hamouda O, Sannes M, et al. Changes in the risk of death after HIV seroconversion compared with mortality in the general population. JAMA 2008;300: 51-59.

4. Gibb DM, Duong T, Tookey PA, et al. Decline in mortality, AIDS, and hospital admissions in perinatally HIV-1 infected children in the United Kingdom and Ireland. Br Med J 2003; 327:1019.

5. Gortmaker SL, Hughes M, Cervia J, et al. Effect of combination therapy including protease inhibitors on mortality among children and adolescents infected with HIV-1. N Engl J Med 2001;345:1522-1528.

6. Judd A, Doerholt K, Tookey PA, et al. Morbidity, mortality, and response to treatment by children in the United Kingdom and Ireland with perinatally acquired HIV infection during 1996-2006: Planning for teenage and adult care. Clin Infect Dis 2007;45:918-924.

7. McDonagh JE, Viner RM. Lost in translation? Between paediatric and adult services. BJM 2006;332:435-436.

8. Watson AR. Non-compliance and transfer from paediatric to adult transplant unit. Pediatr Nephrol 2000;14:469-472.

9. Somerville J. Near misses and disasters in the treatment of grown-up congenital heart patients. R Soc Med 1997;90:124127.

10. Bleyer A. The adolescent and young adult gap in cancer care and outcome. Curr Probl Pediatr Adolesc Health Care 2005;35:182-217.

11. Murphy DA, Belzer M, Durako SJ, Sarr M, Wilson CM, Muenz LR; Adolescent Medicine HIV/AIDS Research Network. Longitudinal antiretroviral adherence among adolescents infected with human immunodeficiency virus. Arch Pediatr Adolesc Med 2005;159:764-770.

12. Kekitiinwa A, Lee KJ, Walker AS, et al. Differences in factors associated with initial growth, CD4 and viral load response to ART in Ugandan and UK/Irish HIV-infected children. $\mathrm{J}$ Acquir Immune Defic Syndr (in press).

13. Catz SL, McClure JB, Jones GN, Brantley PJ. Predictors of outpatient medical appointment attendance among persons with HIV. AIDS Care 1999;11:361-373.

14. Foster C, Melvin D, Portsmouth S, Lyall EGH, Fidler S. Meeting the needs and service development for the longer term follow up of young people with perinatally acquired HIV infection. 12th Annual Conference of the British HIV Association, Brighton 2006 [O32].

15. Department of Health, Child Health and Maternity Services Branch. Transition: getting it right for young people: Improving the transition of young people with long term conditions from children's to adult health services. London: Department of Health. $23^{\text {rd }}$ March2006. www.dh.gov.uk/ en/Publicationsandstatistics/index.htm (Last accessed December 20, 2008.) 
16. Melvin D, Jungmann E, Foster C, et al. Guidance On Transition And Long Term Follow Up Services For Adolescents With HIV Infection Acquired In Infancy. London: Children's HIV Association, 2007.

17. Centers for Disease Control and Prevention. 1994 revised classification system for human immunodeficiency virus infection in children less than 13 years of age. MMWR Morb Mortal Wkly Rep 1994;43:1-10.

18. Cole TJ, Freeman JV, Preece MA: British 1990 growth reference centiles for weight, height, body mass index and head circumference fitted by maximum penalized likelihood. Stat Med 1998;17:407-429.

19. Pillay D, Green H, Matthias R, et al. Estimating HIV-1 drug resistance in antiretroviral-treated individuals in the United Kingdom. J Infect Dis 2005;192:967-973.

20. Johnson VA, Brun-Vezinet F, Clotet B, et al. Update of the drug resistance mutations in HIV-1: 2007. Top HIV Med 2007;15:119-125.

21. Townsend CL, Cliffe S, Tookey PA. Uptake of antenatal HIV testing in the United Kingdom: 2000-2003. J Public Health (Oxf) 2006;28:248-252.

22. Townsend CL, Cortina-Borja M, Peckham CS, de Ruiter A, Lyall $\mathrm{H}$, Tookey PA. Low rates of mother-to-child transmission of HIV following effective pregnancy interventions in the United Kingdom and Ireland, 2000-2006. AIDS 2008;22:973-981.

23. Mollen C, Lavelle J, Hawkins L, Ambrose C, Ruby B. Description of a novel paediatric emergency department-based HIV screening program for adolescents. AIDS Patient Care STDs 2008;22:505-512.

24. Eisenhut M, Sharma V, Kawsar M, Balachandran T. Knowledge of their children's HIV status in HIV-positive mothers attending a genitourinary medicine clinic in the UK. HIV Med 2008;9257-259.

25. Foster C, Waelbrouck A, Peltier A. Adolescents and HIV infection. Curr Opin HIV AIDS 2007;2:431-436.

26. Flynn PM, Rudy BJ, Douglas SD, et al; Pediatric AIDS Clinical Trial Group 381 Study Team. Virologic and immunologic outcomes after 24 weeks in HIV type 1-infected adolescents receiving highly active antiretroviral therapy. J Infect Dis 2004;190:271-279.

27. Mellins CA, Brackis-Cott E, Dolezal C, Abrams EJ. The role of psychosocial and family factors in adherence to antiretroviral treatment in human immunodeficiency virusinfected children. Pediatr Infect Dis J 2004;23:1035-1041.

28. Perno CF, Moyle G, Tsoukas C, Ratanasuwan W, Gatell J, Schechter M. Overcoming resistance to existing therapies in
HIV-infected patients: The role of new antiretroviral drugs. J Med Virol 2008;80:565-576.

29. Kline MW, Rugina S, Ilie M, et al. Long-term follow-up of 414 HIV-infected Romanian children and adolescents receiving lopinavir/ritonavir-containing highly active antiretroviral therapy. Pediatrics 2007;119:e1116-1120.

30. Rutstein RM, Gebo KA, Flynn PM, et al. Immunologic function and virologic suppression among children with perinatally acquired HIV Infection on highly active antiretroviral therapy. Med Care 2005;43(9 Suppl):III15-22.

31. Violari A, Cotton M, Gibb DM, et al. On behalf of the CHER Study team. Early antiretroviral therapy and mortality among HIV-infected infants. N Engl J Med 2008;359:22332244.

32. Welch SB, Gibb D. When should children with HIV infection be started on antiretroviral therapy? PLoS Med 2008;5:e73.

33. Emery, S. Neuhaus JA, Phillips AN, et al. On behalf of the strategies for management of antiretroviral therapy (SMART) Study group. Major clinical outcomes in antiretroviral therapy (ART)-naive participants and in those not receiving ART at baseline in the SMART study. J Infect Dis 2008;197: 1133-1144.

34. Calmy A, Hirschel B, Cooper DA, Carr A. Clinical update: adverse effects of antiretroviral therapy. Lancet 2007,370:1214 .

35. Steele RG, Nelson TD, Cole BP. Psychosocial functioning of children with AIDS and HIV infection: Review of the literature from a socioecological framework. J Dev Behav Pediatr. 2007;28:58-69.

36. Calmy A, Hirschel B, Cooper DA, Carr A. Clinical update: adverse effects of antiretroviral therapy. Lancet 2007,370:1214.

37. Foster CJ, Biggs RL, Melvin D, et al. Neurodevelopmental outcomes in children with HIV infection under 3 years of age. Dev Med Child Neurol 2006;48:677-682.

Address reprint requests to: Caroline Foster, MBBS, MRCPCH

The Family Clinic Imperial College Healthcare NHS Trust 6th floor QEQM, St. Mary's Hospital London W2 1NY United Kingdom

E-mail: drcarolinefoster@btinternet.com 